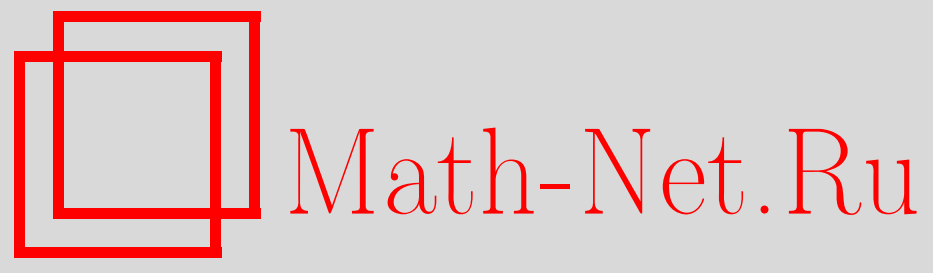

Л. Н. Кривоносов, В. А. Лукьянов, Полное решение уравнений Янга-Миллса для центральносимметрической метрики при наличии электромагнитного поля, Вестн. Сам. гос. техн. ун-та. Сер. Физ.-мат. науки, 2015, номер 3, 462-473

DOI: https://doi.org/10.14498/vsgtu1338

Использование Общероссийского математического портала MathNet.Ru подразумевает, что вы прочитали и согласны с пользовательским соглашением

http://www.mathnet.ru/rus/agreement

Параметры загрузки:

IP : 3.95 .254 .165

26 апреля 2023 г., $16: 47: 21$

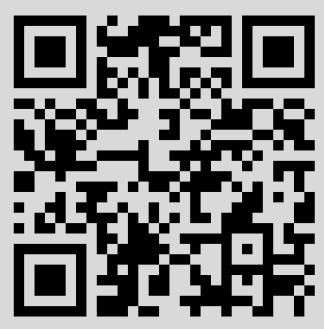




\title{
ПОЛНОЕ РЕШЕНИЕ УРАВНЕНИЙ ЯНГА-МИЛЛСА ДЛЯ ЦЕНТРАЛЬНО-СИММЕТРИЧЕСКОЙ МЕТРИКИ ПРИ НАЛИЧИИ ЭЛЕКТРОМАГНИТНОГО ПОЛЯ
}

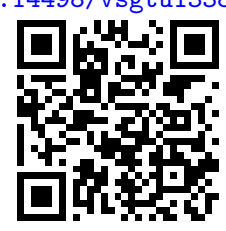

\author{
Л. Н. Кривоносов ${ }^{1}$, В.А. Луквянов ${ }^{2}$ \\ 1 Нижегородский государственный технический университет им. Р. Е. Алексеева, \\ Россия, 603600, Нижний Новгород, ул. Минина, 24. \\ 2 Заволжский филиал Нижегородского государственного \\ технического университета им. Р. Е. Алексеева, \\ Россия, 606520, Нижегородская обл., Заволжье, ул. Павловского, 1а.
}

\begin{abstract}
Аннотация
Ранее авторами было найдено полное решение уравнений Янга-Миллса для центрально-симметрической метрики в 4-мерном пространстве конформной связности без кручения при отсутствии электромагнитного поля. Позже, в другой статье, авторы нашли решения уравнений ЯнгаМиллса для этой же метрики при наличии электромагнитного поля специального вида, предполагая, что его компоненты зависят не от четыpex, а только от двух переменных. Там же авторы провели сравнение получившегося решения с хорошо известным решением Райсснера-Нордстрема и указали причину того, что эти решения не совпадают. В настоящей работе авторы не накладывают никаких предварительных ограничений на компоненты электромагнитного поля. Это сильно усложняет вывод уравнений Янга-Миллса. Тем не менее, все вычислительные трудности были преодолены. Оказалось, что решения этих уравнений всё равно зависят только от двух переменных, и новых решений, кроме полученных ранее, не возникает. Авторы делают вывод, что найдены все решения уравнений Янга-Миллса для центрально-симметрической метрики при наличии произвольного электромагнитного поля, согласованного с уравнениями Янга-Миллса в пространстве без кручения (то есть без источников). Эти решения выражаются через эллиптическую функцию Вейерштрасса.
\end{abstract}

Ключевые слова: кривизна связности, оператор Ходжа, уравнения Эйнштейна, уравнения Максвелла, уравнения Янга-Миллса, центральносимметрическая метрика, эллиптическая функция Вейерштрасса, 4-многообразие конформной связности.

doi: http://dx.doi.org/10.14498/vsgtu1338

(C) 2015 Самарский государственный технический университет.

\section{Образец для цитирования}

Кривоносов Л. Н., Лукьянов В. А. Полное решение уравнений Янга-Миллса для центрально-симметрической метрики при наличии электромагнитного поля // Вестн. Сам. гос. техн. ун-та. Сер. Физ.-мат. науки, 2015. Т. 19, № 3. С. 462-473. doi: $10.14498 / v s g t u 1338$.

Сведения об авторах

Леонид Николаевич Кривоносов (к.ф.-м.н., доц.; l.n.krivonosov@gmail.com; автор, ведущий переписку), доцент, каф. прикладной математики.

Вячеслав Анатольевич Лукъянов, старший преподаватель;охуzt@ya.ru, каф. информатики и общеобразовательных дисциплин. 
Введение. В работе [1] авторы нашли все решения уравнений Янга-Миллса для метрики вида

$$
\psi=-e^{2 \nu} d t^{2}+e^{2 \lambda} d r^{2}+e^{2 \mu}\left(d \theta^{2}+\sigma^{2}(\theta) d \varphi^{2}\right)
$$

где $\lambda, \mu, \nu$ - функции, зависящие только от $r$ и $t$, а функция $\sigma(\theta)$ удовлетворяет уравнению

$$
\frac{d^{2} \sigma}{d \theta^{2}}=-\varkappa \sigma, \quad \varkappa=\text { const },
$$

при нулевом электромагнитном поле. Метрика (1) является небольшим обобщением центрально-симметрической метрики.

При личном общении с одним из авторов настоящей работы (В. А. Лукьяновым) профессор Казанского федерального университета Ю. Г. Игнатьев порекомендовал найти решение уравнения Янга-Миллса для центральносимметрической метрики при наличии электромагнитного поля. Авторы благодарны профессору Ю. Г. Игнатьеву за это предложение. В итоге авторами была опубликована работа [2], в которой найдены решения уравнений ЯнгаМиллса для метрики вида (1) при наличии электромагнитного поля специального вида, компоненты которого в неголономном базисе зависят только от $r$ и $t$. Но в работе [2] авторами была допущена существенная ошибка: промежуточные формулы (1.14) и (1.15) в той работе не должны содержать слагаемых с множителем $\varkappa$. Эта ошибка не повлияла на правильность всех окончательных уравнений и их решений, указанных в [1] при $\frac{1}{\sigma} \frac{d \sigma}{d \theta} \neq$ const, но обесценила обсуждения случая $\frac{1}{\sigma} \frac{d \sigma}{d \theta}=$ const, хотя и в этом случае были получены верные окончательные уравнения.

В настоящей работе авторы сняли всякие предварительные ограничения на компоненты электромагнитного поля. Это сильно усложнило вывод уравнений Янга-Миллса, но оказалось, что в окончательных уравнениях компоненты электромагнитного поля всё равно зависят только от $r$ и $t$, а сами уравнения такие же, как в [2]. Поэтому оказались в силе все решения, указанные в [2], и новых решений не возникает. Таким образом, авторами найдены все решения уравнений Янга-Миллса на 4-многообразии конформной связности без кручения для центрально-симметрической метрики при наличии произвольного электромагнитного поля.

Сделаем следующие замечания.

ЗАмечание 1. Электромагнитное поле, согласованное с уравнениями Янга-Миллса, не содержит источников. Это было доказано авторами в работе [3]. Но еще раньше это было показано в работе [4]. Разница только в том, что в работе [4] сначала были постулированы уравнения Эйнштейна путем наложения некоторых условий, равносильных уравнениям Эйнштейна, на компоненты твисторной связности, а потом выведены уравнения Янга-Миллса. В работе [3] никаких предварительных ограничений на компоненты конформной связности (кроме равенства нулю кручения) не накладывается, а уравнения Эйнштейна возникают в процессе вывода уравнений Янга-Миллса. В работе [4] группа уравнений Янга-Миллса, не сводящаяся к уравнениям Максвелла, записана с помощью тензора Баха [5]. В работе [3] соответствующая группа уравнений была названа уравнениями движения вещества, и в них слагаемые, относящиеся к тензору Баха, специально не выделялись. Если же не обращать в нуль кручение, то электромагнитное поле, согласованное 
с уравнениями Янга-Миллса, уже может иметь источники. Но для этого случае авторы не умеют находить решение уравнения Янга-Миллса.

ЗАмечание 2. Уравнения Янга-Миллса, которые авторы вывели в работе [3] (они же выведены и в работе [4], только к ним надо добавить уравнения Эйнштейна), связывают гравитацию и электромагнетизм совсем не так, как это постулировано Райсснером и Нордстремом. У них тензор электромагнитного поля подставляется в правую часть уравнений Эйнштейна, а в работе [4] этот тензор приравнивается к тензору Баха. Поэтому решения уравнений Янга-Миллса из настоящей статьи ни в коем случае не могут привести к решению Райсснера-Нордстрема уравнений Эйнштейна (это решение можно найти в работе [6]).

ЗАмЕЧАниЕ 3. Авторам настоящей работы известны только две работы, в которых уравнения Янга-Миллса решаются на 4-многообразии конформной связности другими авторами. Это работа [7], в которой найдены решения уравнений Янга-Миллса для метрики Феффермана, и работа [8], где приведено одно чисто временное решение, но оно является частным случаем решений авторов из работы [9]. Сами Янг и Миллс [10] свои уравнения ввели в 1954 году для компактной группы $S U(2)$. С тех пор необозримое количество работ посвящено уравнениям Янга-Миллса и их решениям, но лишь для компактных групп. Авторы же рассматривают 4-многообразия конформной связности с сигнатурой угловой метрики $(-+++)$. Структурная конформная группа при такой сигнатуре некомпактна. K тому же при такой сигнатуре оператор Ходжа $*$ удовлетворяет равенству $*^{2}=-i d$ (а в случае компактных групп $\left.*^{2}=i d\right)$, что не позволяет уравнениям Янга-Миллса иметь автодуальные и антиавтодуальные решения. Поэтому в случае, рассматриваемом авторами настоящей работы, задача решения уравнений Янга-Миллса гораздо труднее, чем для компактных структурных групп. Основная цель авторов построить теорию, объединяющую все 4 фундаментальных взаимодействия. Пока эта задача далека от завершения из-за неумения авторами находить решения уравнения Янга-Миллса при ненулевом кручении.

1. Вывод уравнений Янга-Миллса. В работе [1] указано, что метрику (1) всегда можно привести к виду

$$
\psi=\left(-e^{2 \nu} d t^{2}+e^{2 \lambda} d r^{2}\right)+\left(d \theta^{2}+\sigma^{2}(\theta) d \varphi^{2}\right)
$$

Отправляясь от метрики (3), введем пфаффовы формы

$$
\omega^{1}=e^{\nu} d t, \quad \omega^{2}=e^{\lambda} d r, \quad \omega^{3}=d \theta, \quad \omega^{4}=\sigma(\theta) d \varphi .
$$

Тогда метрика (3) запишется в стандартном виде:

$$
\psi=\eta_{i j} \omega^{i} \omega^{j}
$$

где

$$
\left(\eta_{i j}\right)=\left(\eta^{i j}\right)=\left(\begin{array}{cccc}
-1 & 0 & 0 & 0 \\
0 & 1 & 0 & 0 \\
0 & 0 & 1 & 0 \\
0 & 0 & 0 & 1
\end{array}\right)
$$

есть тензор Минковского. Точкой над буквой будем обозначать частную производную по $t$, штрихом - частную производную по $r$, а производные по $\theta$ и 
$\varphi$ будем записывать символами $\sigma_{\theta},\left(b_{12}\right)_{\varphi}$ и т. д.:

$$
d \omega^{1}=-e^{-\lambda} \nu^{\prime} \omega^{1} \wedge \omega^{2}, \quad d \omega^{2}=e^{-\nu} \dot{\lambda} \omega^{1} \wedge \omega^{2}, \quad d \omega^{3}=0, \quad d \omega^{4}=\frac{\sigma_{\theta}}{\sigma} \omega^{3} \wedge \omega^{4} .
$$

Пфаффовы формы Кристоффеля для квадратичной формы (3) имеют вид

$$
\omega_{1}^{2}=e^{-\lambda} \nu^{\prime} \omega^{1}+e^{-\nu} \dot{\lambda} \omega^{2}, \quad \omega_{3}^{4}=\frac{d \sigma}{d \theta} \frac{1}{\sigma} \omega^{4}, \quad \omega_{1}^{3}=\omega_{1}^{4}=\omega_{2}^{3}=\omega_{2}^{4}=0 .
$$

Внешние 2-формы римановой кривизны квадратичной формы (3) имеют вид

$$
R_{1}^{2}=A \omega^{1} \wedge \omega^{2}, \quad R_{3}^{4}=-\varkappa \omega^{3} \wedge \omega^{4}, \quad R_{1}^{3}=R_{1}^{4}=R_{2}^{3}=R_{2}^{4}=0,
$$

где для краткости введено обозначение

$$
A \stackrel{\text { def }}{=} e^{-2 \lambda}\left(\lambda^{\prime} \nu^{\prime}-\nu^{\prime \prime}-\nu^{\prime 2}\right)+e^{-2 \nu}\left(\ddot{\lambda}-\dot{\lambda} \dot{\nu}+\dot{\lambda}^{2}\right) .
$$

Ненулевые компоненты тензора Риччи $R_{i j}=R_{i j k}^{k}$ и скалярная кривизна $R=$ $=\eta^{i j} R_{i j}$ определяются следующими соотношениями

$$
R_{11}=A, \quad R_{22}=-A, \quad R_{33}=R_{44}=-\varkappa, \quad R=-2 A-2 \varkappa .
$$

Для компонент $b_{j m}$ пфаффовых форм $\omega_{i}=b_{i j} \omega^{j}$ можно вычислить симметрическую часть $b_{(j m)}=b_{j m}+b_{m j}$ из уравнений Эйнштейна $b_{(j m)}=R_{j m}-$ $-\frac{1}{6} R \eta_{j m}[3$, с. 439]. Имеем

$$
\begin{array}{ll}
b_{11}=\frac{1}{3} A-\frac{1}{6} \varkappa, & b_{22}=-\frac{1}{3} A+\frac{1}{6} \varkappa, \\
b_{33}=b_{44}=\frac{1}{6} A-\frac{1}{3} \varkappa, & b_{(j m)}=0 \text { при } j \neq m,
\end{array}
$$

то есть недиагональные элементы кососимметричны.

Уравнения Максвелла имеют вид

$$
d \Phi_{0}^{0}=0, \quad d * \Phi_{0}^{0}=0
$$

[3, с. 440], где

$$
\begin{aligned}
\Phi_{0}^{0}=\frac{1}{2} b_{[i j]} \omega^{j} \wedge \omega^{i}=\frac{1}{2}\left(b_{i j}-b_{j i}\right) \omega^{j} \wedge \omega^{i} & =b_{i j} \omega^{j} \wedge \omega^{i}= \\
= & -2\left(b_{12} \omega^{1} \wedge \omega^{2}+b_{13} \omega^{1} \wedge \omega^{3}+b_{14} \omega^{1} \wedge \omega^{4}+\right. \\
& \left.+b_{23} \omega^{2} \wedge \omega^{3}+b_{24} \omega^{2} \wedge \omega^{4}+b_{34} \omega^{3} \wedge \omega^{4}\right),
\end{aligned}
$$

а *-оператор Ходжа:

$$
\begin{aligned}
* \Phi_{0}^{0}=2\left(-b_{12} \omega^{3} \wedge \omega^{4}+b_{13} \omega^{2} \wedge \omega^{4}-b_{14} \omega^{2}\right. & \wedge \omega^{3}+ \\
& \left.+b_{23} \omega^{1} \wedge \omega^{4}-b_{24} \omega^{1} \wedge \omega^{3}+b_{34} \omega^{1} \wedge \omega^{2}\right) .
\end{aligned}
$$

Уравнения (6) в компонентах принимают соответственно вид

$$
\begin{aligned}
& e^{-\lambda}\left(b_{13}^{\prime}+b_{13} \nu^{\prime}\right)=e^{-\nu}\left(\dot{b_{23}}+b_{23} \dot{\lambda}\right)+\left(b_{12}\right)_{\theta}, \\
& \dot{b_{34}} e^{-\nu}-b_{14} \frac{\sigma_{\theta}}{\sigma}+\frac{\left(b_{13}\right)_{\varphi}}{\sigma}-\left(b_{14}\right)_{\theta}=0, \\
& e^{-\lambda}\left(b_{14}^{\prime}+b_{14} \nu^{\prime}\right)=e^{-\nu}\left(\dot{b_{24}}+b_{24} \dot{\lambda}\right)+\frac{\left(b_{12}\right)_{\varphi}}{\sigma}, \\
& b_{34}^{\prime} e^{-\lambda}-b_{24} \frac{\sigma_{\theta}}{\sigma}+\frac{\left(b_{23}\right)_{\varphi}}{\sigma}-\left(b_{24}\right)_{\theta}=0
\end{aligned}
$$




$$
\begin{aligned}
& e^{-\lambda}\left(b_{24}^{\prime}+b_{24} \nu^{\prime}\right)+\left(b_{34}\right)_{\theta}=e^{-\nu}\left(\dot{b_{14}}+b_{14} \dot{\lambda}\right), \\
& \dot{b_{12}} e^{-\nu}+b_{23} \frac{\sigma_{\theta}}{\sigma}+\left(b_{23}\right)_{\theta}+\frac{\left(b_{24}\right)_{\varphi}}{\sigma}=0, \\
& e^{-\lambda}\left(b_{23}^{\prime}+b_{23} \nu^{\prime}\right)=e^{-\nu}\left(\dot{b_{13}}+b_{13} \dot{\lambda}\right)+\frac{\left(b_{34}\right)_{\varphi}}{\sigma}, \\
& b_{12}^{\prime} e^{-\lambda}+b_{13} \frac{\sigma_{\theta}}{\sigma}+\left(b_{13}\right)_{\theta}+\frac{\left(b_{14}\right)_{\varphi}}{\sigma}=0 .
\end{aligned}
$$

Так как $\omega_{i}=b_{i j} \omega^{j}$, из формул (5) получаем

$$
\begin{aligned}
& \omega_{1}=\left(\frac{1}{3} A-\frac{1}{6} \varkappa\right) \omega^{1}+b_{12} \omega^{2}+b_{13} \omega^{3}+b_{14} \omega^{4}, \\
& \omega_{2}=-b_{12} \omega^{1}+\left(-\frac{1}{3} A+\frac{1}{6} \varkappa\right) \omega^{2}+b_{23} \omega^{3}+b_{24} \omega^{4}, \\
& \omega_{3}=-b_{13} \omega^{1}-b_{23} \omega^{2}+\left(\frac{1}{6} A-\frac{1}{3} \varkappa\right) \omega^{3}+b_{34} \omega^{4}, \\
& \omega_{4}=-b_{14} \omega^{1}-b_{24} \omega^{2}-b_{34} \omega^{3}+\left(\frac{1}{6} A-\frac{1}{3} \varkappa\right) \omega^{4} .
\end{aligned}
$$

Найдём внешние формы конформной кривизны

$$
\Phi_{j}^{i}=R_{j}^{i}+\omega^{i} \wedge \omega_{j}+\eta^{i m} \eta_{j n} \omega_{m} \wedge \omega^{n} \quad \text { и } \quad \Phi_{i}=d \omega_{i}-\omega_{i}^{k} \wedge \omega_{k}
$$

и сразу вычислим преобразования Ходжа [1, с. 352]. При возможности упростим выражения с помощью (7) и (8):

$$
\begin{aligned}
& * \Phi_{3}^{4}=\frac{1}{3}(A+\varkappa) \omega^{1} \wedge \omega^{2}+b_{13} \omega^{2} \wedge \omega^{3}+b_{14} \omega^{2} \wedge \omega^{4}+b_{23} \omega^{1} \wedge \omega^{3}+b_{24} \omega^{1} \wedge \omega^{4}, \\
& * \Phi_{2}^{4}=\frac{1}{6}(A+\varkappa) \omega^{1} \wedge \omega^{3}+b_{12} \omega^{2} \wedge \omega^{3}-b_{14} \omega^{3} \wedge \omega^{4}+b_{23} \omega^{1} \wedge \omega^{2}-b_{34} \omega^{1} \wedge \omega^{4}, \\
& * \Phi_{2}^{3}=-\frac{1}{6}(A+\varkappa) \omega^{1} \wedge \omega^{4}-b_{12} \omega^{2} \wedge \omega^{4}-b_{13} \omega^{3} \wedge \omega^{4}-b_{24} \omega^{1} \wedge \omega^{2}-b_{34} \omega^{1} \wedge \omega^{3} \text {, } \\
& * \Phi_{1}^{4}=-\frac{1}{6}(A+\varkappa) \omega^{2} \wedge \omega^{3}-b_{12} \omega^{1} \wedge \omega^{3}-b_{24} \omega^{3} \wedge \omega^{4}+b_{13} \omega^{1} \wedge \omega^{2}+b_{34} \omega^{2} \wedge \omega^{4} \text {, } \\
& * \Phi_{1}^{3}=\frac{1}{6}(A+\varkappa) \omega^{2} \wedge \omega^{4}+b_{12} \omega^{1} \wedge \omega^{4}-b_{23} \omega^{3} \wedge \omega^{4}-b_{14} \omega^{1} \wedge \omega^{2}+b_{34} \omega^{2} \wedge \omega^{3}, \\
& * \Phi_{1}^{2}=\frac{1}{3}(A+\varkappa) \omega^{3} \wedge \omega^{4}-b_{13} \omega^{1} \wedge \omega^{4}-b_{23} \omega^{2} \wedge \omega^{4}+b_{14} \omega^{1} \wedge \omega^{3}+b_{24} \omega^{2} \wedge \omega^{3} ; \\
& * \Phi_{1}=\left(\dot{b_{12}} e^{-\nu}-\frac{1}{3} e^{-\lambda} A^{\prime}\right) \omega^{3} \wedge \omega^{4}+\left(b_{23} e^{-\lambda} \nu^{\prime}-\dot{b_{13}} e^{-\nu}\right) \omega^{2} \wedge \omega^{4}+ \\
& +\left(\dot{b_{14}} e^{-\nu}-b_{24} e^{-\lambda} \nu^{\prime}\right) \omega^{2} \wedge \omega^{3}+\left(\dot{b_{24}} e^{-\nu}-b_{14} e^{-\lambda} \nu^{\prime}\right) \omega^{1} \wedge \omega^{3}+ \\
& +\left(b_{13} e^{-\lambda} \nu^{\prime}-\dot{b_{23}} e^{-\nu}\right) \omega^{1} \wedge \omega^{4}-\dot{b_{34}} e^{-\nu} \omega^{1} \wedge \omega^{2} \text {, }
\end{aligned}
$$

$$
\begin{aligned}
* \Phi_{2}= & \left(b_{12}^{\prime} e^{-\lambda}-\frac{1}{3} e^{-\nu} \dot{A}\right) \omega^{3} \wedge \omega^{4}+\left(b_{23} e^{-\nu} \dot{\lambda}-b_{13}^{\prime} e^{-\lambda}\right) \omega^{2} \wedge \omega^{4}+ \\
& +\left(b_{14}^{\prime} e^{-\lambda}-b_{24} e^{-\nu} \dot{\lambda}\right) \omega^{2} \wedge \omega^{3}+\left(b_{24}^{\prime} e^{-\lambda}-b_{14} e^{-\nu} \dot{\lambda}\right) \omega^{1} \wedge \omega^{3}+ \\
& +\left(b_{13} e^{-\nu} \dot{\lambda}-b_{23}^{\prime} e^{-\lambda}\right) \omega^{1} \wedge \omega^{4}-b_{34}^{\prime} e^{-\lambda} \omega^{1} \wedge \omega^{2}
\end{aligned}
$$

$$
\begin{aligned}
* \Phi_{3}=\left(b_{12}\right)_{\theta} \omega^{3} & \wedge \omega^{4}-\left(\frac{1}{6} e^{-\nu} \dot{A}+\left(b_{13}\right)_{\theta}\right) \omega^{2} \wedge \omega^{4}+\left(b_{14}\right)_{\theta} \omega^{2} \wedge \omega^{3}- \\
& -\left(\frac{1}{6} e^{-\lambda} A^{\prime}+\left(b_{23}\right)_{\theta}\right) \omega^{1} \wedge \omega^{4}+\left(b_{24}\right)_{\theta} \omega^{1} \wedge \omega^{3}-\left(b_{34}\right)_{\theta} \omega^{1} \wedge \omega^{2},
\end{aligned}
$$




$$
\begin{aligned}
& * \Phi_{4}= \frac{\left(b_{12}\right)_{\varphi}}{\sigma} \omega^{3} \wedge \omega^{4}+\frac{b_{14} \sigma_{\theta}-\left(b_{13}\right)_{\varphi}}{\sigma} \omega^{2} \wedge \omega^{4}+ \\
&+\left(\frac{1}{6} e^{-\nu} \dot{A}+\frac{\left(b_{14}\right)_{\varphi}+b_{13} \sigma_{\theta}}{\sigma}\right) \omega^{2} \wedge \omega^{3}+\left(b_{34}^{\prime} e^{-\lambda}-\left(b_{24}\right)_{\theta}\right) \omega^{1} \wedge \omega^{4}+ \\
& \quad+\left(\frac{1}{6} e^{-\lambda} A^{\prime}+\frac{\left(b_{24}\right)_{\varphi}+b_{23} \sigma_{\theta}}{\sigma}\right) \omega^{1} \wedge \omega^{3}-\frac{\left(b_{34}\right)_{\varphi}}{\sigma} \omega^{1} \wedge \omega^{2} .
\end{aligned}
$$

Найдём внешние 3 -формы $d * \Phi_{i}$ :

$$
\begin{gathered}
d * \Phi_{1}=\left(e^{-2 \nu} b_{14}(\dot{\lambda} \dot{\nu}-\ddot{\lambda})+e^{-2 \lambda} b_{14}\left(\nu^{\prime \prime}-\lambda^{\prime} \nu^{\prime}\right)+\right. \\
\left.+e^{-\lambda-\nu}\left(\dot{b_{24} \nu^{\prime}}-b_{24}^{\prime} \dot{\lambda}\right)+\frac{\left(b_{12}\right)_{\varphi} e^{-\lambda} \nu^{\prime}}{\sigma}\right) \omega^{1} \wedge \omega^{2} \wedge \omega^{3}+ \\
\quad+\left(e^{-2 \nu} b_{13}(\ddot{\lambda}-\dot{\lambda} \dot{\nu})+e^{-2 \lambda} b_{13}\left(\lambda^{\prime} \nu^{\prime}-\nu^{\prime \prime}\right)+\right. \\
\left.+e^{-\lambda-\nu}\left(b_{23}^{\prime} \dot{\lambda}-\dot{b_{23} \nu^{\prime}}\right)-\left(b_{12}\right)_{\theta} e^{-\lambda} \nu^{\prime}\right) \omega^{1} \wedge \omega^{2} \wedge \omega^{4}+ \\
+\left(\frac{1}{3} e^{-\lambda-\nu}\left(A^{\prime} \dot{\lambda}-\dot{A}^{\prime}\right)+b_{12}^{\prime} e^{-2 \lambda} \nu^{\prime}\right) \omega^{1} \wedge \omega^{3} \wedge \omega^{4}+ \\
\quad+\left(e^{-\lambda-\nu} b_{12}^{\prime} \dot{\lambda}+\frac{1}{3} e^{-2 \lambda}\left(A^{\prime} \lambda^{\prime}-A^{\prime \prime}\right)\right) \omega^{2} \wedge \omega^{3} \wedge \omega^{4},
\end{gathered}
$$

$$
\begin{gathered}
d * \Phi_{2}=\left(e^{-2 \nu} b_{24}(\dot{\lambda} \dot{\nu}-\ddot{\lambda})+e^{-2 \lambda} b_{24}\left(\nu^{\prime \prime}-\lambda^{\prime} \nu^{\prime}\right)+\right. \\
\left.+e^{-\lambda-\nu}\left(\dot{b_{14}} \nu^{\prime}-b_{14}^{\prime} \dot{\lambda}\right)+\frac{\left(b_{12}\right)_{\varphi} e^{-\nu} \dot{\lambda}}{\sigma}\right) \omega^{1} \wedge \omega^{2} \wedge \omega^{3}+ \\
\quad+\left(e^{-2 \nu} b_{23}(\ddot{\lambda}-\dot{\lambda} \dot{\nu})+e^{-2 \lambda} b_{23}\left(\lambda^{\prime} \nu^{\prime}-\nu^{\prime \prime}\right)+\right. \\
\left.+e^{-\lambda-\nu}\left(b_{13}^{\prime} \dot{\lambda}-\dot{b_{13} \nu^{\prime}}\right)-\left(b_{12}\right)_{\theta} e^{-\nu} \dot{\lambda}\right) \omega^{1} \wedge \omega^{2} \wedge \omega^{4}+ \\
+\left(e^{-\lambda-\nu} \dot{b_{12}} \nu^{\prime}+\frac{1}{3} e^{-2 \nu}(\dot{A} \dot{\nu}-\ddot{A})\right) \omega^{1} \wedge \omega^{3} \wedge \omega^{4}+ \\
\quad+\left(\frac{1}{3} e^{-\lambda-\nu}\left(\dot{A} \nu^{\prime}-\dot{A}^{\prime}\right)+e^{-2 \nu} \dot{b_{12}} \dot{\lambda}\right) \omega^{2} \wedge \omega^{3} \wedge \omega^{4}
\end{gathered}
$$

$$
\begin{aligned}
d * \Phi_{3}=( & \frac{1}{6} e^{-2 \lambda}\left(A^{\prime \prime}-A^{\prime} \lambda^{\prime}+A^{\prime} \nu^{\prime}\right)+ \\
& \left.+\frac{1}{6} e^{-2 \nu}(\dot{A} \dot{\nu}-\ddot{A}-\dot{A} \dot{\lambda})-\frac{\left(b_{34}\right)_{\varphi} \sigma_{\theta}}{\sigma^{2}}\right) \omega^{1} \wedge \omega^{2} \wedge \omega^{4}+ \\
+ & \left(\frac{\sigma_{\theta}}{6 \sigma} A^{\prime} e^{-\lambda}+b_{23} \varkappa-\frac{\sigma_{\theta}}{\sigma}\left(\left(b_{23}\right)_{\theta}+b_{12} e^{-\nu}\right)\right) \omega^{1} \wedge \omega^{3} \wedge \omega^{4}+ \\
& +\left(\frac{\sigma_{\theta}}{6 \sigma} \dot{A} e^{-\nu}+b_{13} \varkappa-\frac{\sigma_{\theta}}{\sigma}\left(\left(b_{13}\right)_{\theta}+b_{12}^{\prime} e^{-\lambda}\right)\right) \omega^{2} \wedge \omega^{3} \wedge \omega^{4}
\end{aligned}
$$




$$
\begin{aligned}
d * \Phi_{4}= & \left(\frac{1}{6} e^{-2 \lambda}\left(A^{\prime} \lambda^{\prime}-A^{\prime \prime}-A^{\prime} \nu^{\prime}\right)+\frac{1}{6} e^{-2 \nu}(\ddot{A}-\dot{A} \dot{\nu}+\dot{A} \dot{\lambda})\right) \omega^{1} \wedge \omega^{2} \wedge \omega^{3}+ \\
& +\frac{\sigma_{\theta}}{\sigma}\left(b_{34}\right)_{\theta} \omega^{1} \wedge \omega^{2} \wedge \omega^{4}+\left(b_{24} \varkappa-\frac{\sigma_{\theta}}{\sigma}\left(b_{24}\right)_{\theta}\right) \omega^{1} \wedge \omega^{3} \wedge \omega^{4}+ \\
& +\left(b_{14} \varkappa-\frac{\sigma_{\theta}}{\sigma}\left(b_{14}\right)_{\theta}\right) \omega^{2} \wedge \omega^{3} \wedge \omega^{4} .
\end{aligned}
$$

При $i=1$ получим первое уравнение Янга-Миллса [1, с. 352]:

$$
d * \Phi_{1}+\omega_{i} \wedge * \Phi_{1}^{i}-* \Phi_{i} \wedge \omega_{1}^{i}-* \Phi_{0}^{0} \wedge \omega_{1}=0
$$

С помощью полученных выше выражений запишем его в компонентах и получим четыре уравнения:

$$
\begin{aligned}
& b_{12} b_{24}+b_{13} b_{34}=0, \quad b_{12} b_{23}-b_{14} b_{34}=0 \\
& e^{-\lambda-\nu}\left(\dot{A} \nu^{\prime}+A^{\prime} \dot{\lambda}-\dot{A}^{\prime}\right)+12 b_{13} b_{23}+12 b_{14} b_{24}=0, \\
& e^{-2 \lambda}\left(A^{\prime} \lambda^{\prime}-A^{\prime \prime}\right)+\frac{1}{2}\left(\varkappa^{2}-A^{2}\right)+e^{-2 \nu} \dot{A} \dot{\lambda}+ \\
& \quad+6\left(\left(b_{12}\right)^{2}+\left(b_{13}\right)^{2}+\left(b_{14}\right)^{2}+\left(b_{23}\right)^{2}+\left(b_{24}\right)^{2}+\left(b_{34}\right)^{2}\right)=0 .
\end{aligned}
$$

При $i=2$ получаем еще три уравнения:

$$
\begin{aligned}
& b_{12} b_{14}+b_{23} b_{34}=0, \quad b_{12} b_{13}-b_{24} b_{34}=0 \\
& e^{-2 \nu}(\dot{A} \dot{\nu}-\ddot{A})+\frac{1}{2}\left(A^{2}-\varkappa^{2}\right)+e^{-2 \lambda} A^{\prime} \nu^{\prime}+ \\
& \quad+6\left(\left(b_{13}\right)^{2}+\left(b_{14}\right)^{2}+\left(b_{23}\right)^{2}+\left(b_{24}\right)^{2}-\left(b_{12}\right)^{2}-\left(b_{34}\right)^{2}\right)=0
\end{aligned}
$$

При $i=3$ будем иметь только два новых уравнения:

$$
\begin{aligned}
& b_{23} b_{24}-b_{13} b_{14}=0, \\
& e^{-2 \lambda}\left(A^{\prime \prime}-A^{\prime} \lambda^{\prime}+A^{\prime} \nu^{\prime}\right)+e^{-2 \nu}(\dot{A} \dot{\nu}-\ddot{A}-\dot{A} \dot{\lambda})+A^{2}-\varkappa^{2}+ \\
& \quad+12\left(\left(b_{13}\right)^{2}-\left(b_{14}\right)^{2}-\left(b_{23}\right)^{2}+\left(b_{24}\right)^{2}-\left(b_{12}\right)^{2}-\left(b_{34}\right)^{2}\right)=0 .
\end{aligned}
$$

При $i=4$ добавится лишь уравнение

$$
\begin{aligned}
& e^{-2 \lambda}\left(A^{\prime \prime}-A^{\prime} \lambda^{\prime}+A^{\prime} \nu^{\prime}\right)+e^{-2 \nu}(\dot{A} \dot{\nu}-\ddot{A}-\dot{A} \dot{\lambda})+A^{2}-\varkappa^{2}+ \\
& +12\left(\left(b_{14}\right)^{2}+\left(b_{23}\right)^{2}-\left(b_{13}\right)^{2}-\left(b_{24}\right)^{2}-\left(b_{12}\right)^{2}-\left(b_{34}\right)^{2}\right)=0 .
\end{aligned}
$$

Уравнения (10)-(13) получаются в таком виде только после использования (для упрощения) формул (7), (8) и (4). Например, в уравнении (9) приравнивание к нулю коэффициента при $\omega^{1} \wedge \omega^{2} \wedge \omega^{3}$ первоначально приводило к соотношению

$$
\begin{aligned}
e^{-2 \nu} b_{14}\left(\dot{\lambda} \dot{\nu}-\ddot{\lambda}-\dot{\lambda}^{2}\right) & +e^{-2 \lambda}\left(\nu^{\prime \prime}-\nu^{\prime} \lambda^{\prime}-b_{14}^{\prime} \nu^{\prime}\right)+\frac{1}{\sigma}\left(b_{12}\right)_{\varphi} e^{-\lambda} \nu^{\prime}+ \\
& +e^{-\lambda-\nu}\left(\dot{b_{24}} \nu^{\prime}+b_{24} \nu^{\prime} \dot{\lambda}\right)+b_{14} A-4 b_{12} b_{24}-4 b_{13} b_{34}=0 .
\end{aligned}
$$

Теперь, если вместо $A$ подставить его выражение из (4), то будем иметь

$$
e^{-\lambda} \nu^{\prime}\left(e^{-\nu}\left(\dot{b_{24}}+b_{24} \dot{\lambda}\right)-e^{-\lambda}\left(b_{14}^{\prime}+b_{14} \nu^{\prime}\right)+\frac{\left(b_{12}\right)_{\varphi}}{\sigma}\right)-4 b_{12} b_{24}-4 b_{13} b_{34}=0
$$


Наконец, используя одно из уравнений (7), приходим к первому уравнению (10).

Итак, все уравнения Янга-Миллса свелись к (7), (8) и (10)-(13), где величина $A$ вычисляется по формуле (4).

2. Отыскание решений системы уравнений (7), (8) и (10)-(13). Выпишем короткие уравнения из (10)-(11):

$$
\begin{array}{ll}
b_{12} b_{13}-b_{24} b_{34}=0, & b_{12} b_{23}-b_{14} b_{34}=0 \\
b_{12} b_{14}+b_{23} b_{34}=0, & b_{12} b_{24}+b_{13} b_{34}=0 .
\end{array}
$$

Эти равенства можно рассматривать как линейную однородную систему уравнений с неизвестными $b_{13}, b_{14}, b_{23}, b_{24}$. Определитель ее основной матрицы равен $\left(\left(b_{12}\right)^{2}+\left(b_{34}\right)^{2}\right)^{2}$. В зависимости от того, равен он нулю или нет, возникают две возможности.

Первый случай: $\left(b_{12}\right)^{2}+\left(b_{34}\right)^{2} \neq 0$. Это означает, что $b_{13}=b_{14}=b_{23}=$ $=b_{24}=0$. Уравнения (7) и (8) сводятся к тому, что $b_{12}$ и $b_{34}$ являются константами. Из (10)-(13) получим всего три независимых уравнения:

$$
\begin{aligned}
& \dot{A} \nu^{\prime}+A^{\prime} \dot{\lambda}-\dot{A}^{\prime}=0 \\
& e^{-2 \lambda}\left(A^{\prime} \lambda^{\prime}-A^{\prime \prime}\right)+e^{-2 \nu} \dot{A} \dot{\lambda}+\frac{1}{2}\left(K^{2}-A^{2}\right)=0 \\
& e^{-2 \nu}(\dot{A} \dot{\nu}-\ddot{A})+e^{-2 \lambda} A^{\prime} \nu^{\prime}+\frac{1}{2}\left(A^{2}-K^{2}\right)=0
\end{aligned}
$$

где

$$
K \stackrel{\text { def }}{=} \sqrt{\varkappa^{2}+12\left(b_{12}\right)^{2}+12\left(b_{34}\right)^{2}}=\text { const. }
$$

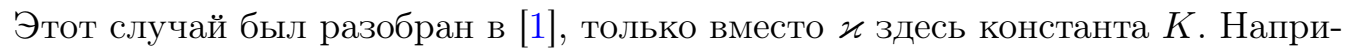
мер, при $\nu=0$ имеем

$$
A(t, r)=12 \wp\left(t+s(r), \frac{K^{2}}{12}, \alpha\right), \quad e^{\lambda}=\dot{A} s^{\prime}(r),
$$

где $\alpha=$ const, $s(r)$ - произвольная функция.

Второй случай: $b_{12}=b_{34}=0$. Система (10)-(13) примет вид

$$
\begin{aligned}
e^{-\lambda-\nu}\left(\dot{A} \nu^{\prime}+A^{\prime} \dot{\lambda}-\dot{A}^{\prime}\right)+12 b_{13} b_{23}+12 b_{14} b_{24}=0 & \\
e^{-2 \lambda}\left(A^{\prime} \lambda^{\prime}-A^{\prime \prime}\right)+ & \frac{1}{2}\left(\varkappa^{2}-A^{2}\right)+e^{-2 \nu} \dot{A} \dot{\lambda}+ \\
& +6\left(\left(b_{13}\right)^{2}+\left(b_{14}\right)^{2}+\left(b_{23}\right)^{2}+\left(b_{24}\right)^{2}\right)=0 \\
e^{-2 \nu}(\dot{A} \dot{\nu}-\ddot{A})+ & \frac{1}{2}\left(A^{2}-\varkappa^{2}\right)+e^{-2 \lambda} A^{\prime} \nu^{\prime}+ \\
& +6\left(\left(b_{13}\right)^{2}+\left(b_{14}\right)^{2}+\left(b_{23}\right)^{2}+\left(b_{24}\right)^{2}\right)=0 \\
b_{23} b_{24}-b_{13} b_{14}= & 0 \\
e^{-2 \lambda}\left(A^{\prime \prime}-A^{\prime} \lambda^{\prime}+\right. & \left.A^{\prime} \nu^{\prime}\right)+e^{-2 \nu}(\dot{A} \dot{\nu}-\ddot{A}-\dot{A} \dot{\lambda})+A^{2}-\varkappa^{2}+ \\
& +12\left(\left(b_{13}\right)^{2}-\left(b_{14}\right)^{2}-\left(b_{23}\right)^{2}+\left(b_{24}\right)^{2}\right)=0 \\
e^{-2 \lambda}\left(A^{\prime \prime}-A^{\prime} \lambda^{\prime}+\right. & \left.A^{\prime} \nu^{\prime}\right)+e^{-2 \nu}(\dot{A} \dot{\nu}-\ddot{A}-\dot{A} \dot{\lambda})+\left(A^{2}-\varkappa^{2}\right)+ \\
& +12\left(\left(b_{14}\right)^{2}+\left(b_{23}\right)^{2}-\left(b_{13}\right)^{2}-\left(b_{24}\right)^{2}\right)=0
\end{aligned}
$$

Последние два уравнения равносильны уравнениям

$$
e^{-2 \lambda}\left(A^{\prime \prime}-A^{\prime} \lambda^{\prime}+A^{\prime} \nu^{\prime}\right)+e^{-2 \nu}(\dot{A} \dot{\nu}-\ddot{A}-\dot{A} \dot{\lambda})+A^{2}-\varkappa^{2}=0,
$$




$$
\left(b_{14}\right)^{2}+\left(b_{23}\right)^{2}-\left(b_{13}\right)^{2}-\left(b_{24}\right)^{2}=0 .
$$

Разность последнего уравнения (14) и уравнения (15) дает (17). Первое уравнение (16) совместно с уравнением (18) имеет только следующее решение:

$$
b_{23}=\varepsilon b_{13}, \quad b_{24}=\varepsilon b_{14}, \quad \varepsilon= \pm 1 .
$$

В итоге вся система уравнений Янга-Миллса для 2-го случая сводится к системе

$$
\begin{gathered}
\left(b_{13} e^{\nu}\right)^{\prime}=\varepsilon\left(b_{13} e^{\lambda}\right)^{\cdot}, \quad\left(b_{14} e^{\nu}\right)^{\prime}=\varepsilon\left(b_{14} e^{\lambda}\right) ; \\
\left(b_{13}\right)_{\varphi}=\left(\sigma b_{14}\right)_{\theta}, \quad\left(b_{14}\right)_{\varphi}+\left(\sigma b_{13}\right)_{\theta}=0 ; \\
e^{-\lambda-\nu}\left(\dot{A} \nu^{\prime}+A^{\prime} \dot{\lambda}-\dot{A}^{\prime}\right)+\varepsilon L=0, \\
e^{-2 \nu}(\dot{A} \dot{\nu}-\ddot{A})+\frac{1}{2}\left(A^{2}-\varkappa^{2}\right)+e^{-2 \lambda} A^{\prime} \nu^{\prime}+L=0, \\
e^{-2 \lambda}\left(A^{\prime} \lambda^{\prime}-A^{\prime \prime}\right)+\frac{1}{2}\left(\varkappa^{2}-A^{2}\right)+e^{-2 \nu} \dot{A} \dot{\lambda}+L=0,
\end{gathered}
$$

где $L \stackrel{\text { def }}{=} 12\left(\left(b_{13}\right)^{2}+\left(b_{14}\right)^{2}\right)$.

В первом уравнении (20) величина $e^{-\lambda-\nu}\left(\dot{A} \nu^{\prime}+A^{\prime} \dot{\lambda}-\dot{A}^{\prime}\right)$ зависит только от $t$ и $r$, следовательно, $L=12\left(\left(b_{13}\right)^{2}+\left(b_{14}\right)^{2}\right)$ также зависит только $t$ и $r$. Отсюда, дифференцируя по $\theta$ и $\varphi$, получаем

$$
b_{13}\left(b_{13}\right)_{\theta}+b_{14}\left(b_{14}\right)_{\theta}=0, \quad b_{13}\left(b_{13}\right)_{\varphi}+b_{14}\left(b_{14}\right)_{\varphi}=0 .
$$

Подставляем сюда вместо $\left(b_{13}\right)_{\varphi}$ и $\left(b_{14}\right)_{\varphi}$ выражения из последних уравнений (19) и приходим к системе

$$
\begin{aligned}
& b_{13}\left(b_{14}\right)_{\theta}-b_{14}\left(b_{13}\right)_{\theta}=0, \\
& b_{14}\left(b_{14}\right)_{\theta}+b_{13}\left(b_{13}\right)_{\theta}=0
\end{aligned}
$$

линейных однородных уравнений с неизвестными $\left(b_{14}\right)_{\theta}$ и $\left(b_{13}\right)_{\theta}$ и определителем основной матрицы $\left(b_{13}\right)^{2}+\left(b_{14}\right)^{2}$. Если он равен нулю, то это означает отсутствие электромагнитного поля, и мы приходим к ситуации, разобранной в [1]. Если он не равен нулю, то

$$
\left(b_{14}\right)_{\theta}=\left(b_{13}\right)_{\theta}=0
$$

и последние два уравнения (19) запишутся в виде

$$
\left(b_{13}\right)_{\varphi}=b_{14} \sigma_{\theta}, \quad\left(b_{14}\right)_{\varphi}+b_{13} \sigma_{\theta}=0 .
$$

Так как $b_{14}$ и $b_{13}$ не зависят от $\theta$, величина $\sigma_{\theta}$ также не должна зависеть от $\theta$, то есть $\sigma_{\theta}=C=$ const, $\sigma_{\theta \theta}=0$. Из (2) получаем $\varkappa=0$. В этом случае заменой переменных

$$
x=\frac{\sigma}{C} \cos C \varphi, \quad y=\frac{\sigma}{C} \sin C \varphi
$$

форма $d \theta^{2}+\sigma^{2}(\theta) d \varphi^{2}$ сводится к $d x^{2}+d y^{2}$, то есть $\sigma=1$. Тогда из (22) следует, что $\left(b_{13}\right)_{\varphi}=\left(b_{14}\right)_{\varphi}=0$. Учитывая $(21)$, приходим к случаю, когда $b_{i j}$ зависят только от $t$ и $r$, то есть к ситуации, разобранной в [2]. Система (19), (20) станет такой же, как в [2, формулы 2.14]. 
Выводы. Уравнения Янга-Миллса, полученные нами в первом случае, когда $\left(b_{12}\right)^{2}+\left(b_{34}\right)^{2} \neq 0$, полностью совпадают с теми, что были найдены в [2], так как $b_{12}$ и $b_{34}$ оказываются зависящими только от $t$ и $r$. Их общее решение выражается через эллиптическую функцию Вейерштрасса. Второй случай, когда $b_{12}=b_{34}=0$, возможен лишь при $\varkappa=0$ (в этом случае метрика (1) центрально-симметрической не называется). Здесь снова получается такая же система уравнений, как и в [2]. Хотя она и является вполне интегрируемой, найти ее общее решение в элементарных функциях авторам не удалось.

\section{ORCIDs}

Кривоносов Леонид Николаевич: http://orcid.org/0000-0002-3533-9595

Лукьянов Вячеслав Анатольевич: http://orcid.org/0000-0002-7294-0232

\section{БИБЛИОГРАФИЧЕСКИЙ СПИСОК}

1. Кривоносов Л. Н., Лукьянов В. А. Полное решение уравнений Янга-Миллса для центрально-симметрической метрики // Журн. СФУ. Сер. Матем. и физ., 2011. Т.4, № 3. С. $350-362$.

2. Кривоносов Л. Н., Лукьянов В. А. Решение уравнений Янга-Миллса для центральносимметрической метрики при наличии электромагнитного поля // Пространство, время и фундаментальные взаимодействия, 2013. № 3. С. 54-63.

3. Кривоносов Л. Н., Лукьянов В. А. Связь уравнений Янга-Миллса с уравнениями Эйнштейна и Максвелла // Журн. СФУ. Сер. Матем. и физ., 2009. Т. 2, № 4. С. 432-448.

4. Меркулов С. А. Твисторная связность и конформная гравитация // ТМФ, 1984. Т. 60, № 2. C. 311-316.

5. Bach R. Zur Weylschen Relativitätstheorie und der Weylschen Erweiterung des Krümmungstensorbegriffs // Math. Z., 1921. vol.9, no.1. pp. 110-135. doi:10.1007/ bf01378338.

6. Владимиров Ю. С. Геометрофизика. М.: Бином, 2010. 536 с.

7. Korzyński M., Lewandowski J. The normal conformal Cartan connection and the Bach tensor // Class. Quantum Grav., 2003. vol. 20, no. 16. pp. 3745-3764, arXiv: gr-qc/0301096. doi : $10.1088 / 0264-9381 / 20 / 16 / 314$.

8. Трунев А. П. Моделирование метрики адронов на основе уравнений Янга-Миллса // Научный журнал КубГАУ, 2012. № 84(10). C. 1-14, http://ej.kubagro.ru/2012/10/ pdf/68.pdf.

9. Merkulov S. A. A conformally invariant theory of gravitation and electromagnetism // Class. Quantum Grav., 1984. vol. 1, no.4. pp. 349-354. doi: 10.1088/0264-9381/1/4/007.

10. Krivonosov L. N., Lukyanov V. A. Purely time-dependent solutions to the Yang-Mills equations on a 4-dimensional manifold with conformal torsion-free connection // J. Sib. Fed. Univ. Math. Phys., 2013. vol. 6, no. 1. pp. 40-52.

11. Yang C. N., Mills R. L. Conservation of Isotopic Spin and Isotopic Gauge Invariance // Phys. Rev., 1954. vol. 96, no. 1. pp. 191-195. doi: 10.1103/physrev.96.191.

Поступила в редакцию 26/IX/2014; в окончательном варианте - 02/III/2015; принята в печать - 08/IV/2015. 
Vestn. Samar. Gos. Techn. Un-ta. Ser. Fiz.-mat. nauki

[J. Samara State Tech. Univ., Ser. Phys. \& Math. Sci.], 2015, vol. 19, no. 3, pp. $462-473$

ISSN: 2310-7081 (online), 1991-8615 (print)

doi: http://dx.doi.org/10.14498/vsgtu1338

MSC: 83E99, 83C22

\title{
THE COMPLETE SOLUTION OF THE YANG-MILLS EQUATIONS FOR CENTRALLY SYMMETRIC METRIC IN THE PRESENCE OF ELECTROMAGNETIC FIELD
}

\author{
L. N. Krivonosov ${ }^{1}$, V. A. Luk'yanov ${ }^{2}$ \\ 1 Nizhny Novgorod State Technical University, \\ 24, Minina st., Nizhnii Novgorod, 603600, Russian Federation. \\ 2 Zavolzhskij Branch of Nizhny Novgorod State Technical University, \\ 1a, Pavlovskogo st., Zavolzh'e, Nizhegorodskaya obl., 606520, Russian Federation.
}

\begin{abstract}
Previously, we found the complete solution of Yang-Mills equations for a centrally symmetric metric in 4-dimensional space of conformal torsion-free connection in the absence of the electromagnetic field. Later, in another article, we found a solution of the Yang-Mills equations for the same metric in the presence of an electromagnetic field of a special type, suggesting that its components depend not on the four, but only on two variables. There we compared the resulting solutions with the well-known Reissner-Nordstrom solution and indicated the reason why these solutions do not match. In this paper, we do not impose any prior restrictions on the components of the electromagnetic field. This greatly complicates the derivation of the YangMills equations. However, all computational difficulties were overcome. It turned out that the solutions of these equations all the same depend only on two variables and new solutions, in addition to previously obtained, do not arise. Consequently, we have found all the solutions of the Yang-Mills equations for a centrally symmetric metric in the presence of an arbitrary electromagnetic field, agreed with the Yang-Mills equations in the torsionfree space (i.e., without sources). These solutions are expressed in terms of the Weierstrass elliptic function.
\end{abstract}

Keywords: curvature of the connection, Hodge operator, Einstein equations, Maxwell's equations, Yang-Mills equations, centrally symmetric metric, Weierstrass elliptic function, 4-manifold with conformal connection.

doi: http://dx.doi.org/10.14498/vsgtu1338

(C) 2015 Samara State Technical University.

Please cite this article in press as:

Krivonosov L. N., Luk'y an ov V. A. The complete solution of the Yang-Mills equations for centrally symmetric metric in the presence of electromagnetic field, Vestn. Samar. Gos. Tekhn. Univ., Ser. Fiz.-Mat. Nauki [J. Samara State Tech. Univ., Ser. Phys. \& Math. Sci.], 2015, vol. 19, no. 3, pp. 462-473. doi: 10.14498/vsgtu1338. (In Russian)

\section{Authors Details:}

Leonid N. Krivonosov (Cand. Phys. \& Math. Sci.; 1.n.krivonosov@gmail.com; Corresponding Author), Associate Professor, Dept. of Applied Mathematics.

Vyacheslav A. Luk'yanov, Senior Lecturer, Dept. of Computer Science and General Disciplines. 


\section{ORCIDs}

Leonid N. Krivonosov: http://orcid.org/0000-0002-3533-9595

Vyacheslav A. Luk'yanov: http://orcid.org/0000-0002-7294-0232

\section{REFERENCES}

1. Krivonosov L. N, Luk'yanov V. A. The full decision of Young-Mills equations for the centralsymmetric metrics, J. Sib. Fed. Univ. Math. Phys., 2011, vol.4, no.3, pp. 350-362 (In Russian).

2. Krivonosov L. N, Luk'yanov V. A. Solution of Yang-Mills equations for central-cymmetric metric in the presence of electromagnetic field, Prostranstvo, vremia $i$ fundamental'nye vzaimodeistviia [Space, Time, and Fundamental Interactions], 2013, no. 3, pp. 54-63 (In Russian).

3. Krivonosov L. N, Luk'yanov V. A. Connection of Young-Mills Equations with Einstein and Maxwell's Equations, J. Sib. Fed. Univ. Math. Phys., 2009, vol. 2, no. 4, pp. 432-448 (In Russian).

4. Merkulov S. A. Twistor connection and conformal gravitation, Theoret. and Math. Phys., 1984, vol. 60, no. 2, pp. 842-845. doi: 10.1007/BF01018984.

5. Bach R. Zur Weylschen Relativitätstheorie und der Weylschen Erweiterung des Krümmungstensorbegriffs, Math. Z., 1921, vol.9, no.1, pp. 110-135. doi:10.1007/ bf 01378338 .

6. Vladimirov Yu. S. Geometrofizika [Geometrophysics]. Moscow, Binom, 2010, 536 pp. (In Russian)

7. Korzyński M., Lewandowski J. The normal conformal Cartan connection and the Bach tensor, Class. Quantum Grav., 2003, vol. 20, no. 16, pp. 3745-3764, arXiv: gr-qc/0301096. doi : $10.1088 / 0264-9381 / 20 / 16 / 314$.

8. Trunev A. P. Hadrons metrics simulation on the Yang-Mills equations, Nauchnyi zhurnal KubGAU, 2012, no.84(10), pp. 1-14 (In Russian), http://ej.kubagro.ru/2012/10/pdf/ 68.pdf.

9. Merkulov S. A. A conformally invariant theory of gravitation and electromagnetism, Class. Quantum Grav., 1984, vol. 1, no. 4, pp. 349-354. doi: 10.1088/0264-9381/1/4/007.

10. Krivonosov L. N., Lukyanov V. A. Purely time-dependent solutions to the Yang-Mills equations on a 4-dimensional manifold with conformal torsion-free connection, J. Sib. Fed. Univ. Math. Phys., 2013, vol. 6, no. 1, pp. 40-52.

11. Yang C. N., Mills R. L. Conservation of Isotopic Spin and Isotopic Gauge Invariance, Phys. Rev., 1954, vol.96, no.1, pp. 191-195. doi: 10.1103/physrev.96.191.

Received 26/IX/2014;

received in revised form $02 / \mathrm{III} / 2015$;

accepted 08/IV/2015. 